\title{
Ambulante integrative onkologische Rehabilitation: Ein Pilotprojekt in der Schweiz
}

\author{
Manfred E. Heim ${ }^{a, b}$ Thomas Schröter ${ }^{a} \quad$ Elke Wünnenberg ${ }^{a}$ \\ Brigitte Werner $^{\mathrm{a}} \quad$ Martina Bösch ${ }^{\mathrm{a}}$ Gabriela Keller $^{\mathrm{c}}$
}

${ }^{\mathrm{a} G e s u n d h e i t s z e n t r u m ~ B o d e n s e e, ~ K l i n i k ~ S o k r a t e s, ~ G u ̈ t t i n g e n, ~ S c h w e i z ~}$

bUniversität Göttingen, Deutschland

'SHI Haus der Homöopathie, Zug, Schweiz

\section{Schlüsselwörter}

Rehabilitation · Integrative Onkologie · Ambulante onkologische Rehabilitation · Pilotprojekt

\section{Zusammenfassung}

Hintergrund: In der Versorgung von Krebspatienten spielt die Rehabilitation nach Abschluss der Akuttherapie eine wichtige Rolle, da häufig funktionelle Störungen und eine psychosoziale Belastung bestehen. Um den Patientenbedürfnissen gerecht zu werden, ist ein ganzheitlicher, biopsychosozialer Therapieansatz erforderlich. Patienten und Methode: In einer Pilotstudie wurde die Durchführbarkeit und Wirksamkeit einer ambulanten Rehabilitation über 8 Wochen bei Tumorpatienten geprüft. Das komplexe Therapieprogramm beinhaltete neben Therapien zur Verbesserung der funktionalen Gesundheit komplementäre Massnahmen wie Musiktherapie und Homöopathie. Der Erfolg der Rehabilitation wurde aus ärztlicher Sicht nach 8 Wochen durch eine Kontrolluntersuchung beurteilt. Am Anfang und Ende der Rehabilitation wurde der subjektive Patientenerfolg durch validierte Fragebögen erhoben. Ergebnisse: Das Pilotprojekt wurde von den Patienten positiv bewertet. In den Fragebögen konnte eine Besserung von Distress, Angst, Depression und Fatigue am Ende der Rehabilitation nachgewiesen werden. Schlussfolgerungen: Die onkologische Rehabilitation mit Einbeziehung von komplementären Therapien und Homöopathie wurde von den Patienten der Pilotstudie positiv bewertet. Subjektive Belastungen besserten sich im Verlauf. Weitere Studien mit grösseren Patientenzahlen und längeren Verlaufskontrollen sind geplant, um die Nachhaltigkeit des Konzepts zu überprüfen.

\section{Keywords}

Rehabilitation · Integrative oncology · Ambulatory oncological rehabilitation · Pilot project

\section{Summary \\ Ambulatory Integrative Oncological Rehabilitation: \\ A Pilot Project in Switzerland}

Background: In the care for cancer patients, rehabilitation following an acute therapy is of great importance because of frequent functional deficits and psychosocial distress. To satisfy the patients' needs a holistic biopsychosocial treatment is required. Patients and Method: In a pilot study we assessed the feasibility and efficiency of an 8-week ambulatory rehabilitation in cancer patients. The complex treatment program included therapies to improve the functional health as well as complementary therapies, such as music therapy and homeopathy. The rehabilitation outcome after 8 weeks was evaluated by a medical control examination. The patient-reported outcome was documented by validated questionnaires at the beginning and at the end of the rehabilitation. Results: The patients assessed the pilot project to be beneficial. By means of the questionnaires an improvement of distress, anxiety, depression and fatigue was found at the end of the rehabilitation. Conclusions: Oncological rehabilitation including complementary therapies and homeopathy was positively rated by the patients in this pilot study. Subjective distress improved in the course of rehabilitation. Further studies with more patients and a longer follow-up are planned to test the durability of the treatment concept.

\section{Mots-clés}

Réadaptation - Oncologie intégrative - Réadaptation oncologique ambulatoire · Projet pilote

\section{Résumé}

Réadaptation oncologique intégrative ambulatoire: un projet pilote en Suisse

Contexte: La réadaptation des patients atteints de cancer une fois le traitement aigu achevé joue un rôle important dans leur prise en charge, étant donné l'existence fréquente de troubles fonctionnels et d'une charge psychosociale. Afin de satisfaire les besoins des patients, une approche thérapeutique biopsychosociale globale est nécessaire. Patients et méthode: Dans une étude pilote, la faisabilité et l'efficacité d'une réadaptation ambulatoire ont été examinées pendant 8 semaines chez des patients atteints de tumeur. Outre les traitements visant à améliorer la santé fonctionnelle, le programme thérapeutique complexe comportait des mesures complémentaires telles que la musicothérapie et l'homéopathie. Le succès de la réadaptation a été évalué du point de vue médical après 8 semaines par un examen de contrôle. Le succès subjectif du patient a été relevé au début et à la fin de la réadaptation au moyen de questionnaires validés. Résultats: Le projet pilote a reçu une évaluation positive de la part des patients.

\section{KARGER \\ Fax +49761 4520714 Information@Karger.d} www.karger.com
Prof. Dr. med. Manfred E. Heim

Gesundheitszentrum Bodensee, Klinik Sokrates

Im Park 3, 8594 Güttingen, Schweiz

me.heim@klinik-sokrates.ch 
Les questionnaires ont permis de démontrer une amélioration en termes de détresse, d'angoisse, de dépression et de fatigue à la fin de la réadaptation. Conclusions: La réadaptation oncologique intégrant des traitements complémentaires et l'homéopathie ont reçu une évaluation positive de la part des patients de l'étude pilote. Le stress subjectif s'est amélioré durant le suivi. D'autres études incluant un plus grand nombre de patients et un prolongement des contrôles de suivi sont prévues afin de vérifier la durabilité du concept.

\section{Einleitung}

Jedes Jahr erkranken etwa 35000 Menschen in der Schweiz an Krebs. Aufgrund verbesserter Therapien sind die Heilungschancen gestiegen (ca. 50\%) und der Anteil der Überlebenden nach einer Krebserkrankung nimmt kontinuierlich zu. Da die Behandlungen komplexer, langwieriger und nebenwirkungsreicher geworden sind, ist der Bedarf an Nachsorge und Rehabilitation gestiegen. Die Verweildauer im Akutkrankenhaus wird zunehmend kürzer, während die Lebenserwartung und die Lebensarbeitszeit zunehmen [1]. In Deutschland zeigt sich der zunehmende Bedarf an medizinischen Rehabilitationsleistungen in der Onkologie am Anstieg der Bewilligungen der Deutschen Rentenversicherung Bund für die stationäre Rehabilitation von Tumorpatienten trotz Budgetierung auf 163564 Patienten im Jahr 2010 [2]. Geht man davon aus, dass der Rehabilitationsbedarf von Krebskranken in der Schweiz nicht geringer sein wird, so wäre mit mindestens 12600 stationären onkologischen Rehabilitationen zu rechnen. Dieser theoretische Bedarf wird zurzeit nicht abgedeckt, und auch fokale ambulante Therapien dürften zur adäquaten Versorgung nicht ausreichen.

Tab. 1. Patienten

\begin{tabular}{ll}
\hline Anzahl & $\mathrm{n}=11$ \\
Alter (mittel), Jahre & $62(46-75)$ \\
Tumortherapie beendet & $<6$ Monate $\mathrm{n}=7$ \\
& $>6$ Monate $\mathrm{n}=4$ \\
Heimatort & Thurgau $\mathrm{n}=7$ \\
& St. Gallen $\mathrm{n}=1$ \\
& Süddeutschland $\mathrm{n}=3$ \\
Tumordiagnosen & Mammakarzinom $(\mathrm{n}=3)$ \\
& Ovarialkarzinom $(\mathrm{n}=2)$ \\
& Rektumkarzinom \\
& malignes Melanom \\
& Harnblasenkarzinom \\
& Nierenzellkarzinom \\
& Bronchialkarzinom \\
& Plasmozytom \\
& Lymphödem Arm bzw. Beine, \\
& Schulter-Arm-Syndrom, Polyneuro- \\
pathie, Rezidivängste, Anpassungs- \\
Typische Rehabilitations- \\
diagnosen & Ernährungsstörungen, Schmerzsyndrom \\
&
\end{tabular}

Die Krebsliga Schweiz hat in den Kantonen Freiburg, Wallis und Zürich ein ambulantes interprofessionelles Netzwerk für onkologische Rehabilitation gefördert (20082011). Eine wissenschaftliche Auswertung soll erfolgen [3].

Tumorpatienten haben ein hohes Interesse an ganzheitlich ausgerichteten und komplementären Therapiemassnahmen, die in der Schweiz eine besondere Stellung einnehmen. In einem Modellprojekt wird eine patientenorientierte Rehabilitation mit komplementären und ressourcenaktivierenden Therapien kombiniert.

Ziel der Untersuchung war es, die Durchführbarkeit einer ambulanten onkologischen Rehabilitation im ländlichen Bereich der Ostschweiz zu prüfen. Daneben sollte der Therapieerfolg nach Abschluss der Rehabilitation durch ärztliche Kontrolluntersuchungen und Patientenbefragungen (patient-reported outcome) mit validierten Fragebögen festgestellt werden.

\section{Patienten und Methode}

Das Gesundheitszentrum Bodensee in Güttingen/Thurgau wurde von der Stiftung Sokrates für Gesundheit, Erziehung und Bildung mit dem Ziel gegründet, in einer integrativen Medizin patientenorientiert schulmedizinische Therapien mit komplementären Massnahmen zu vereinen. Entsprechend einem biopsychosozialen Krankheitsmodell wird die Wiederherstellung oder Verbesserung der körperlichen, seelischen und geistigen Gesundheit angestrebt, wobei besonderer Wert auf die Gesundheitsförderung, im Sinne einer Salutogenese, gelegt wird. In einem Ambulatorium werden zurzeit ambulante Therapien durchgeführt. Ein Klinikneubau mit 33 Betten und grosszügigen Therapieräumen direkt am Bodensee wird Ende 2012 fertiggestellt werden. Indikationsschwerpunkt ist die Rehabilitation von Tumorpatienten.

In dem Pilotprojekt wurde entsprechend den Rahmenempfehlungen für die ambulante onkologische Rehabilitation ein interdisziplinäres Therapieprogramm über 8 Wochen zusammengestellt $[4,5]$.

Vor Beginn des Programms wurden alle Patienten von einem Rehabilitationsmediziner und einem internistischen Onkologen untersucht. Gemeinsam mit dem Patienten wurden anschliessend Rehabilitationsdiagnose, Rehabilitationsbedarf und Rehabilitationsziele festgelegt.

Voraussetzung für die Teilnahme am Programm waren eine ausreichende Rehabilitationsfähigkeit und Mobilität. Neben dem Gruppentherapieprogramm wurden die erforderlichen Einzeltherapien mit den Patienten abgestimmt.

Das Curriculum bestand aus 8 wöchentlichen Gruppentherapien und 8 weiteren Tagen mit Einzeltherapien. Während des Gruppentages wurden die folgenden therapeutischen Elemente eingesetzt:

- Bewegungstherapie (aerobes Ausdauertraining, isometrisches Krafttraining, Achtsamkeitspraxis);

- Yoga;

- themenzentrierte Gesprächsgruppe mit Musiktherapie (Gruppenthemen, unter anderem Ressourcen erkunden, Ich und die anderen, «Zwiegespräch», Resonanzmethode, Achtsamkeitsübungen, Integration und Abschiednehmen);

- Physiotherapie (unter anderem Franklin-Methode [6]);

- Vorträge mit Diskussion (Tumorentstehung, Tumortherapie, Umgehen mit Angst und Stress, Schmerz, Homöopathie, Patientenkompetenz unter anderem);

- Entspannung und Meditation mit Visualisierungen.

Je nach Indikation wurden zusätzlich Einzeltherapien angewandt (Musiktherapie, Physiotherapie, Meridian- und Farbtherapie nach Christel Heidemann [7], Homöopathie, Ernährungsberatung, Magnetfeldtherapie). Bei 7 Patienten wurde begleitend eine individuelle homöopathische Therapie verordnet. 5 Patienten, bei denen die Tumortherapie längere Zeit 
zurücklag, wurden konstitutionell behandelt. 2 Patienten, die die Chemotherapie erst vor Kurzem beendet hatten, wurden symptomatisch behandelt.

Um die Nachhaltigkeit der Rehabilitation zu verbessern, erhielten die Patienten nach jedem Therapietag Hausaufgaben, Merkblätter, Tagebücher sowie CDs mit Vorträgen und Entspannungsanleitungen. Die Patientengruppe war sehr heterogen zusammengesetzt (Tab. 1).

Die Auswertung des ambulanten Reha-Programms erfolgte durch klinische Kontrolluntersuchungen nach 4 und nach 8 Wochen. Der klinische Erfolg aus ärztlicher Sicht (clinical benefit) wurde mit der CGI-Skala (Clinical Global Improvement Scale) zwischen «1 = sehr viel gebessert» und «7 = sehr viel schlechter» bewertet.

Zusätzlich wurden die Patienten gebeten, am Anfang und Ende des Programms validierte Fragebögen zur Belastung (Distress-Thermometer [8]), zu Angst und Depression (Hospital Anxiety and Depression Scale, HADS [9]) und zur tumorassoziierten Fatigue (Multidimensional Fatigue Inventory, MFI [10]) auszufüllen.

Der Erfolg der Rehabilitation wurde einerseits durch das Arzturteil (Besserung von Symptomatik und klinischem Befund), andererseits durch subjektive Veränderungen aus der Sicht der Patienten bestimmt. Auf längere Nachuntersuchungen wurde aufgrund des Pilotcharakters der Studie verzichtet.

\section{Ergebnisse}

Alle Patienten nahmen sehr motiviert am ambulanten Rehabilitationsprogramm teil. Eine Patientin mit progredienter Peritonealkarzinose musste wegen eines notwendigen Spitalaufenthalts nach 4 Wochen ausscheiden; eine weitere Patientin konnte aus logistischen Gründen nur am Gruppentherapieprogramm teilnehmen.

Aus ärztlicher Sicht wurden am Ende des Therapieprogramms deutliche Besserungen von Funktionsstörungen, wie z.B. Armbeweglichkeit, Lymphödem und Polyneuropathie, beobachtet. Auf der CGI-Skala wurde im Mittel eine Veränderung von 2,8 (2 = viel gebessert; 3 = gering gebessert) festgestellt.

Tab. 2. Ergebnisse der Patientenfragebögen im Verlauf (prä/post)

\begin{tabular}{lccl}
\hline & Vor Reha & Nach Reha & Differenz \\
\hline Distress-Thermometer (0-10) & 5,7 & 5,4 & $-0,3$ \\
HADS Angst (0-21) & 9,2 & 8,4 & $-0,8$ \\
Depression (0-21) & 5,9 & 5,3 & $-0,6$ \\
MFI (General Fatigue, 0-100) & 57 & 49 & -8 \\
\hline
\end{tabular}

Tab. 3. Patientenbewertung der Rehabilitationstherapien (Mittelwerte: $0-5 ; 5=$ sehr gut; 4 = gut)

\begin{tabular}{ll}
\hline Gruppentherapie & \\
Themenzentrierte Gruppe & 4,11 \\
Bewegungstherapiegruppe & 4,67 \\
Physiotherapiegruppe & 4,56 \\
Entspannungsgruppe & 4,78 \\
Vorträge & 4,21 \\
Einzeltherapien & \\
Physiotherapie & 4,75 \\
Musiktherapie & 4,78 \\
Ärztliche Beratung & 4,33 \\
Ernährungsberatung & 4,50 \\
Homöopathie & 4,50 \\
Klangwoge («Vitabalance») & 4,29 \\
\hline
\end{tabular}

Ambulante onkologische Rehabilitation
Die validierten Fragebögen wurden von allen Patienten am Anfang sowie am Ende des Pilotprojekts ausgefüllt (Tab. 2). Auffällig war eine relativ hohe Belastung durch Angst und Fatigue vor der Therapie. Auch die mittlere Distress-Belastung war erhöht. Für alle Parameter war eine Besserung am Therapieende festzustellen.

Am Abschluss des Programms wurden die Patienten gebeten, in einem Fragebogen alle Therapiemassnahmen einzeln zu beurteilen und ihre Meinung als Freitext abzugeben (Tab. 3). Insgesamt bewerteten alle Patienten das Reha-Programm als hilfreich und würden es anderen Betroffenen weiterempfehlen. Zitat: «Die Reha hat mir mehr Lebensfreude und Besserung im Umgang mit Ängsten gebracht» oder auch «Ich habe mehr Achtsamkeit und Gelassenheit im Alltag gelernt».

\section{Diskussion}

In einem ambulanten achtwöchigen Pilotprojekt zur Rehabilitation von Krebspatienten konnte die Durchführbarkeit und Wirksamkeit des integrativen Therapieansatzes nachgewiesen werden («proof of concept»). Einschränkend sind allerdings die geringe Anzahl der behandelten Patienten und die fehlenden Kontrolluntersuchungen im weiteren Verlauf zu erwähnen. Aus rehabilitationswissenschaftlichen Studien ist bekannt, dass die Effekte der Rehabilitation häufig nicht anhaltend sind [11].

Die Therapiedichte war im Vergleich zur ambulanten Rehabilitation, die analog zur dreiwöchigen stationären Rehabilitation durchgeführt wurde, relativ gering [5, 12]. Aus diesem Grund ist eine Intensivierung der Rehabilitation durch ein dreiwöchiges Programm mit täglichen Anwendungen nach Fertigstellung der Klinik Sokrates Ende 2012 geplant.

Der biopsychosoziale Ansatz der Rehabilitation zur Verbesserung der funktionalen Gesundheit $[13,14]$ wurde im beschriebenen Pilotprojekt durch komplementäre Therapien ergänzt. Diese Integration kam Patientenbedürfnissen entgegen und hat durch intensive Diskussion im Therapeutenteam zu hoher Akzeptanz geführt. Die Nutzung künstlerischer Therapien, hier Musiktherapie, im psychoonkologischen Kontext hat sich als wirksame Erweiterung der Therapiemöglichkeiten gezeigt [15]. Dem individuellen Bedürfnis der Patienten nach Spiritualität wurde durch Meditationen, Visualisierungen und Beratung im Einzelgespräch entsprochen.

In der Studie bestand das Angebot der homöopathischen Mitbetreuung, das von 7 Patienten genutzt wurde. Die gemeinsame Betreuung der Patienten setzt eine hohe Kooperationsbereitschaft im Therapeutenteam voraus. Teamsitzungen und telefonische Absprachen trugen dazu bei, ein gemeinsames ganzheitliches Therapiekonzept zu entwickeln, das den Patienten kommuniziert werden konnte. 
Zur Anwendung der Homöopathie bei Krebspatienten liegen nur wenige wissenschaftliche Untersuchungen vor. Einzelne Studien zeigen günstige Effekte auf die Nebenwirkungen von Tumortherapien und auf die Lebensqualität der Patienten [16]. In den publizierten Studien wurden ernsthafte unerwünschte Wirkungen oder Interaktionen mit konventionellen Tumortherapien nicht berichtet.

In der Physiotherapie wurden gute Erfahrungen mit der Franklin-Methode [6], die Achtsamkeitsübungen für Körpergefühl und Visualisierungen einbezieht, gemacht. Für die Behandlung von Narbenbeschwerden wurde die Farbmeridiantherapie nach Christel Heidemann [7] mit subjektiv gutem Erfolg eingesetzt.

Naturgemäss lässt sich bei einem komplexen Therapieprogramm nicht entscheiden, welches Therapieelement für die Besserung der Beschwerden und des Befindens der Patienten verantwortlich ist. Die Rückmeldungen der Teilnehmer bestärken uns jedoch in der Annahme, dass das hier erprobte ganzheitliche Therapiekonzept den Wünschen und Bedürfnissen der Patienten entspricht und wirksam ist.
Eine Weiterentwicklung und ein Ausbau der komplexen onkologischen Rehabilitation ist bei der derzeitigen Unterversorgung in der Schweiz dringend notwendig [17]. In einer randomisierten Studie (CAMON, Case Management in Oncology Rehabilitation) soll in der Schweiz geprüft werden, ob die Versorgung nach der Tumorbehandlung durch «case management» verbessert werden kann [3].

Eine systematische Übersicht über Studien zur Auswirkung von "case management» bei Tumorpatienten konnte jedoch keinen Nachweis für die Effektivität erbringen [18]. Wir halten daher ein komplexes Rehabilitationsprogramm nach Abschluss der Akutbehandlung ("Anschlussheilbehandlung»), das von einem qualifizierten Therapeutenteam eingesetzt wird, für patientenorientierter und wirkungsvoller.

Es wäre wünschenswert, in künftigen Studien mit grösseren Patientenzahlen unterschiedliche Konzepte zur Rehabilitation von Krebspatienten vergleichend $\mathrm{zu}$ evaluieren.

\section{Disclosure Statement}

Die Autoren versichern, dass kein Interessenkonflikt besteht.

\section{Literatur}

1 Heim ME: Onkologische Rehabilitation: Wege zurück ins Leben. Leading Opinions Hämatologie/Onkologie 2008;1:8-12.

2 Deutsche Rentenversicherung Bund: Reha-Bericht 2012. Berlin, Deutsche Rentenversicherung Bund, 2012, pp 1-96.

3 Bachmann-Mettler I, Steurer-Stey C, Senn O, Wang M, Bardheci K, Rosemann T: Case management in oncology rehabilitation: the effect of case management on the quality of life in patients with cancer after one year of ambulant rehabilitation. A study protocol for a randomized controlled clinical trial in oncology rehabilitation. Trials 2011;12:103.

4 Deutsche Rentenversicherung Bund: Rahmenkonzept zur medizinischen Rehabilitation in der gesetzlichen Rentenversicherung. Berlin, Deutsche Rentenversicherung Bund, 2007, pp 1-66.

5 Bundesarbeitsgemeinschaft für Rehabilitation (BAR): Rahmenempfehlungen zur ambulanten onkologischen Rehabilitation. Frankfurt/M., Bundesarbeitsgemeinschaft für Rehabilitation, 2004, pp 1-59.
6 Franklin E: Locker sein macht stark. München, Kösel, 1998.

7 Heidemann C: Meridiantherapie - Die Wiederherstellung der Ordnung lebendiger Prozesse. Badenweiler, Eigenverlag, 1998.

$\checkmark 8$ Mehnert A, Müller D, Koch U: Die deutsche Version des NCCN Distress-Thermometers Empirische Prüfung eines Screening-Instruments zur Erfassung psychosozialer Belastung bei Krebspatienten. Z Psychiatr Psychol Psychother 2006;54:213-223.

$\checkmark 9$ Herrmann C: International experiences with the Hospital Anxiety and Depression Scale a review of validation data and clinical results. J Psychosom Res 1997;42:17-41.

10 Smets E, Garssen B, Bonke B, De Haes J: The Multidimensional Fatigue Inventory psychometric qualities of an instrument to assess fatigue. J Psychom Res 1995;39:315-325.

11 Heim ME, Kunert S, Özkan I: Effects of inpatient rehabilitation on health-related quality of life in breast cancer patients. Onkologie 2001;24:268-272.
Bartsch HH: Stellenwert und Perspektiven der Rehabilitation onkologischer Patienten in Deutschland. Onkologie 2001;24(suppl 1):6873.

13 Schuntermann MF: Einführung in die ICF, ed 2. Landsberg am Lech, ecomed Medizin, 2007.

14 DIMDI: ICF - Internationale Klassifikation der Funktionsfähigkeit, Behinderung und Gesundheit. Neu-Isenburg, MMI, 2006.

15 Rose JP, Brandt K, Weis J: Musiktherapie in der Onkologie. Eine kritische Analyse zum Stand der Forschung. Psychother Psych Med 2004;54:457-470.

16 Rostock M, Saller R: Komplementäre Therapieverfahren in der Onkologie: Homöopathie. Onkologe 2009;15:1243-1250.

17 Hewitt M, Greenfield S, Stovall E (eds): From Cancer Patient to Cancer Survivor: Lost in Transition. Washington DC, National Academies Press, 2006.

18 Wulff CN, Thygesen M, Sondergaard J, Vedsted P: Case management used to optimize cancer care pathways: a systematic review. BMC Health Serv Res 2008;8:227. 